\title{
Single Crystal, Luminescent Carbon Nitride Nanosheets Formed by Spontaneous Dissolution
}

\author{
Thomas S. Miller, $^{\dagger}$ Theo M. Suter, ${ }^{\dagger}$ Andrew M. Telford, ${ }^{\ddagger}$ Loren Picco, $^{\S}$ Oliver D. Payton, ${ }^{\S}$
} Freddie Russell-Pavier, ${ }^{\S}$ Patrick L. Cullen, ${ }^{\|}$Andrea Sella, ${ }^{\dagger}$ Milo S. P. Shaffer, ${ }^{\perp}$ Jenny Nelson, ${ }^{\ddagger}$ Vasiliki Tileli, ${ }^{\#}$ Paul F. McMillan, ${ }^{* \dagger}{ }^{\dagger}$ and Christopher A. Howard ${ }^{*}, \|_{\odot}$

${ }^{\dagger}$ Department of Chemistry, Christopher Ingold Laboratory, University College London, 20 Gordon Street, London WC1H OAJ, United Kingdom

${ }^{\ddagger}$ Department of Physics and Centre for Plastic Electronics, Imperial College London, London SW7 2BW, United Kingdom

${ }^{\S}$ Interface Analysis Centre, H. H. Wills Physics Laboratory, University of Bristol, Tyndall Avenue, Bristol BS8 1TL, United Kingdom

"Department of Physics \& Astronomy, University College London, London WC1E 6BT, United Kingdom

${ }^{\perp}$ Department of Chemistry, Imperial College London, Exhibition Road, London SW7 2AZ, United Kingdom

\#Institute of Materials, École Polytechnique Fédérale de Lausanne, CH-1015 Lausanne, Switzerland

\section{Supporting Information}

ABSTRACT: A primary method for the production of $2 \mathrm{D}$ nanosheets is liquid-phase delamination from their 3D layered bulk analogues. Most strategies currently achieve this objective by significant mechanical energy input or chemical modification but these processes are detrimental to the structure and properties of the resulting $2 \mathrm{D}$ nanomaterials. Bulk poly(triazine imide) (PTI)-based carbon nitrides are layered materials with a high degree of crystalline order. Here, we demonstrate that these semiconductors are spontaneously soluble in select polar aprotic solvents, that is, without any chemical or physical intervention. In contrast to more aggressive exfoliation strategies, this thermodynamically driven

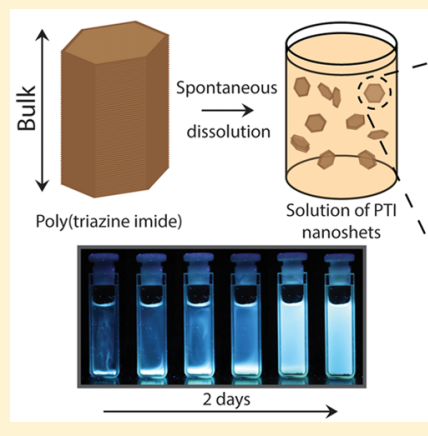

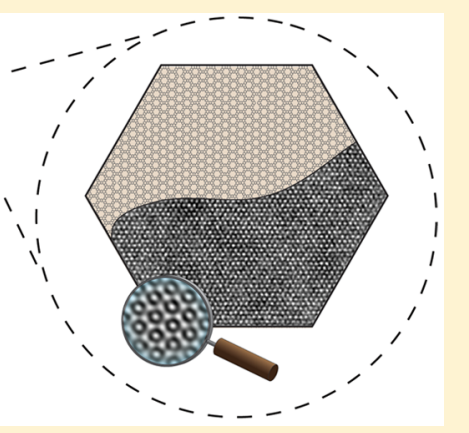
dissolution process perfectly maintains the crystallographic form of the starting material, yielding solutions of defect-free, hexagonal 2D nanosheets with a well-defined size distribution. This pristine nanosheet structure results in narrow, excitationwavelength-independent photoluminescence emission spectra. Furthermore, by controlling the aggregation state of the nanosheets, we demonstrate that the emission wavelengths can be tuned from narrow UV to broad-band white. This has potential applicability to a range of optoelectronic devices.

KEYWORDS: Nanomaterial, exfoliation, poly(triazine imide), photoactive, two-dimensional material, solution

$\mathrm{T}^{\mathrm{k}}$ he rapidly expanding catalogue of functional two-dimensional (2D) materials exhibits a range of remarkable physical and chemical properties. Such nanomaterials can be exploited as individual nanostructures or as multilayer assemblies, networks, and heterostructures in many different scientific and technological contexts. ${ }^{1-4}$ The $2 \mathrm{D}$ nanomaterials can be formed by direct bottom-up synthesis, by micromechanical exfoliation, and through a range of liquid phase approaches. $^{5-13}$ Each strategy has its advantages and disadvantages, but liquid phase exfoliation from layered crystalline precursors offers several benefits, most particularly the potential to prepare and then subsequently manipulate the nanomaterials at industrial scales. However, current liquid phase methods typically rely on aggressive chemical or physical processes to separate the layers, followed by ultracentrifugation to remove large aggregates, making the processes difficult to scale-up. Moreover, such routes typically result in metastable suspensions of fragmented, physically damaged, or chemically modified nanosheets. ${ }^{5,7,8,12-14}$ In an ideal scenario, layers of the parent material would separate spontaneously within the liquid to form pristine solvated nanosheets that maintain their original in-plane structure with the desirable properties intact. Such solutions could then be used to efficiently print, interleave, assemble, and embed the sheets into functional membranes, films, coatings or composites. ${ }^{1,2}$

Layered clay minerals represent a class of materials that can exhibit spontaneous, thermodynamically driven swelling and eventual delamination upon solvent contact. ${ }^{15-17}$ In these systems, the sheets possess a permanent net charge, achieved by isomorphic substitution of atoms within the layers and

Received: March 31, 2017

Revised: June 15, 2017

Published: July 5, 2017 
accompanying intercalation of charge-balancing interlayer cations. For naturally uncharged layered materials, including graphite, true dissolution into polar aprotic solvents can also be achieved by introducing charges onto the layers via intercalation of ions. ${ }^{18-20}$ This promotes spontaneous exfoliation of the layers to produce true solutions of charged anionic nanosheets within the solvent. ${ }^{20}$

Polymeric or graphitic carbon nitrides ( $\mathrm{gCNs}$ ) are being developed for applications including catalysis, photocatalysis, and energy storage/conversion. ${ }^{11,21-29}$ Many of these applications exploit the fact that gCN materials are chemically robust semiconductors with bandgaps ranging between approximately 2.2-2.8 eV (ref 21). The poly(triazine imide) (PTI) form of carbon nitride has attracted specific interest because of its high degree of crystallinity (Figure 1a). ${ }^{30,31}$ These slightly buckled,
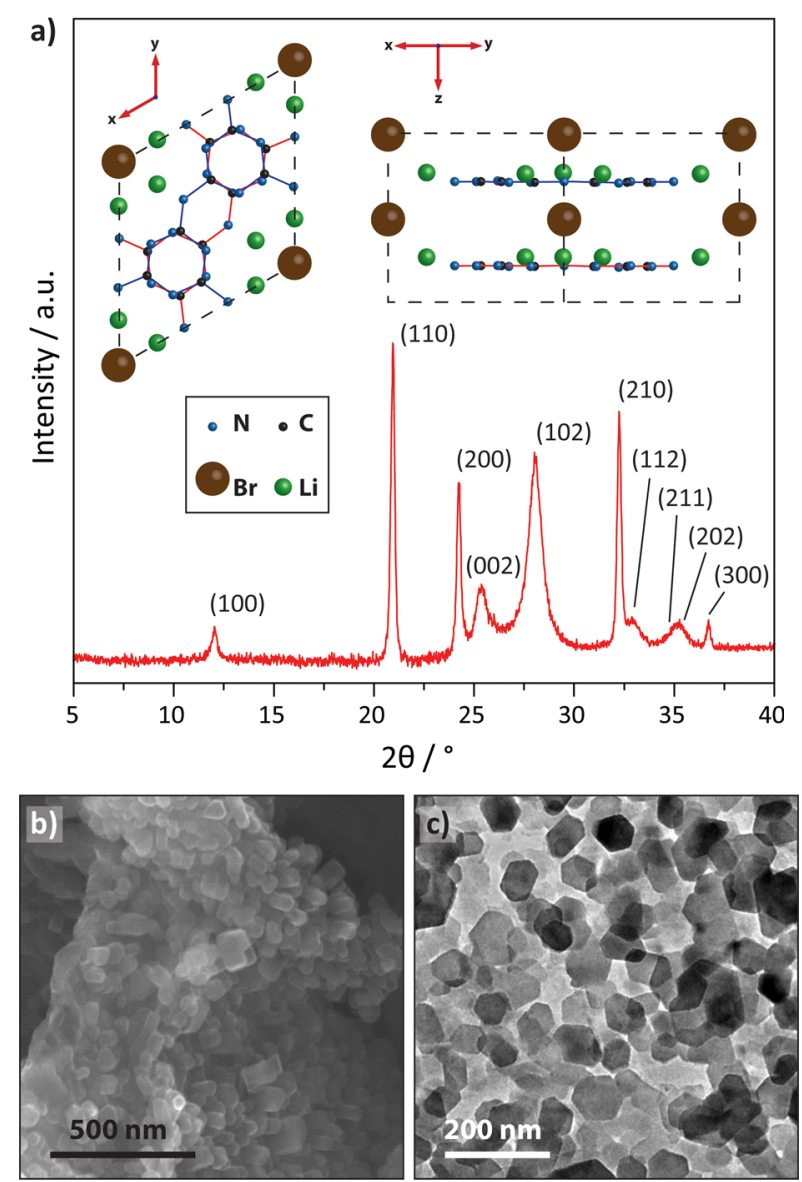

Figure 1. Structure and characterization of crystalline PTI-LiBr. (a) Powder XRD pattern of crystalline PTI $\cdot \mathrm{LiBr}$, indexed according to a $\mathrm{P6}_{3} \mathrm{~cm}$ space group lattice. ${ }^{31,35}$ Inset shows one unit cell of a PTI.LiBr, assuming the average crystal structure presented by Wirnhier et al. ${ }^{31}(\mathrm{~b})$ SEM image of an aggregate of hexagonal prismatic PTI.LiBr crystallites. (c) TEM image of a bundle of as-synthesized hexagonal PTI-LiBr crystallites.

planar layers typically have a $\mathrm{C} / \mathrm{N}$ ratio near $2: 3$ and consist of triazine $\left(\mathrm{C}_{3} \mathrm{~N}_{3}\right)$ rings linked via imido $(-\mathrm{NH}-,-\mathrm{N}=)$ groups, causing the appearance of voids within the sheets. These voids are typically decorated internally with $\mathrm{H}^{+}$or $\mathrm{Li}^{+}$ions and $\mathrm{Cl}^{-}$or $\mathrm{Br}^{-}$counterions that occupy sites within the void or held between the layers $^{30,31}$ (Figure 1). The solid compound forms hexagonal prismatic crystallites (Figure 1).
Liquid-based strategies used to exfoliate layered gCN materials, thus far, have employed aggressive mechanical or chemical methods. ${ }^{32,33}$ An early study introduced potassium into $\mathrm{PTI} \cdot \mathrm{LiBr}$ via vapor intercalation, followed by reaction with water, ${ }^{7,34}$ resulting in nanomaterials that were tens of layers thick and not did not resemble the hexagonal crystallites characteristic of the bulk precursor. Exfoliation of PTI materials via prolonged sonication in water has also been reported. ${ }^{25}$ This approach yielded few-layer nanosheets at concentrations up to $0.2 \mathrm{mg} / \mathrm{mL}$, which were accessible following multiple ultracentrifugation steps to remove unexfoliated material and aggregates from the nanosheet suspensions. Here, we demonstrate that crystalline $\mathrm{PTI} \cdot \mathrm{LiBr}$ can spontaneously dissolve into aprotic polar solvents to form true solutions containing defect-free, crystalline, semiconducting 2D nanosheets.

Bulk PTI.LiBr was produced by a condensation reaction from dicyandiamide (DCDA) in a eutectic $\mathrm{LiBr}-\mathrm{KBr}$ molten salt (Supporting Information Section SI 1, methods). Powder X-ray diffraction (XRD) analysis confirmed the crystalline nature of the layered material (Figure 1a), as shown previously. ${ }^{31}$ Scanning and transmission electron microscopy (SEM, TEM) revealed the hexagonal prismatic structure of the crystallites, as dictated by their underpinning $\mathrm{Pb}_{3} \mathrm{~cm}$ symmetry (Figure $1 \mathrm{~b}, \mathrm{c}$ ). ${ }^{31}$ These crystallites commonly have heights (through plane) $>150 \mathrm{~nm}$, that is, $>400$ layers thick (Figure $1 \mathrm{~b}$ ). A histogram of the lateral dimensions (from two parallel edges across the hexagonal plane) shows the crystallites are typically between 30 and $165 \mathrm{~nm}$ across with an average of $66 \mathrm{~nm}$ (Figure S1).

Graphitic carbon nitrides are typically considered as insoluble compounds. ${ }^{36}$ However, herein we demonstrate the dissolution of PTI $\mathrm{LiBr}$ in a range of polar aprotic organic solvents including $\mathrm{N}$-methylpyrrolidone (NMP), N,N-dimethylformamide (DMF), and dimethyl sulfoxide (DMSO). Upon the careful addition of solvent to the solid sample, a change in the color of the liquid was observed over time (Figure 2, Figure S2), as species dissolve into the solvent. The visibility of the dissolution process was enhanced when under UV-light illumination. Not all solvents could solubilize the material (Supporting Information Section SI 2 , solvent systems), for example, when using ethanol no dissolution was observed, even after 7 days (Figure 2). We utilized differences in the properties of the efficacious solvents to enhance our ability to collect data for different techniques (Supporting Information Section SI 1, dissolution of PTI).

The concentration of dissolved solute was determined from the supernatant by thermogravimetric analysis (TGA, Supporting Information Section SI 1 and Figure S3). After 7 days, the PTI dissolved in DMSO (shown in Figure 2) was found to have a concentration of $0.8 \pm 0.05 \mathrm{mg} \mathrm{mL}^{-1}$.

To investigate the structure and morphology of the solute, aliquots were carefully removed from the uppermost part of the solution after 7 days and dropped onto holey-carbon covered copper grids for TEM evaluation, or freshly cleaved mica substrates for atomic force microscopy (AFM) measurements, followed by removal of solvent (methods).

Figure 3a and Figure S4 show high-resolution (HR) TEM images of typical PTI. LiBr nanosheets deposited following dissolution in NMP. The carbon nitride nanosheet (Figure 3a) is atomically intact up to its well-defined edges and the hexagonal shape and lateral dimensions are close to those of the precursor bulk crystals (Figure 3f). To enhance the contrast of the different atomic columns of the 2D PTI sheets, the defocus condition was adjusted accordingly (Figure 3b,c). Coherent atomic column contrast is preserved throughout the nanosheet with no evidence 


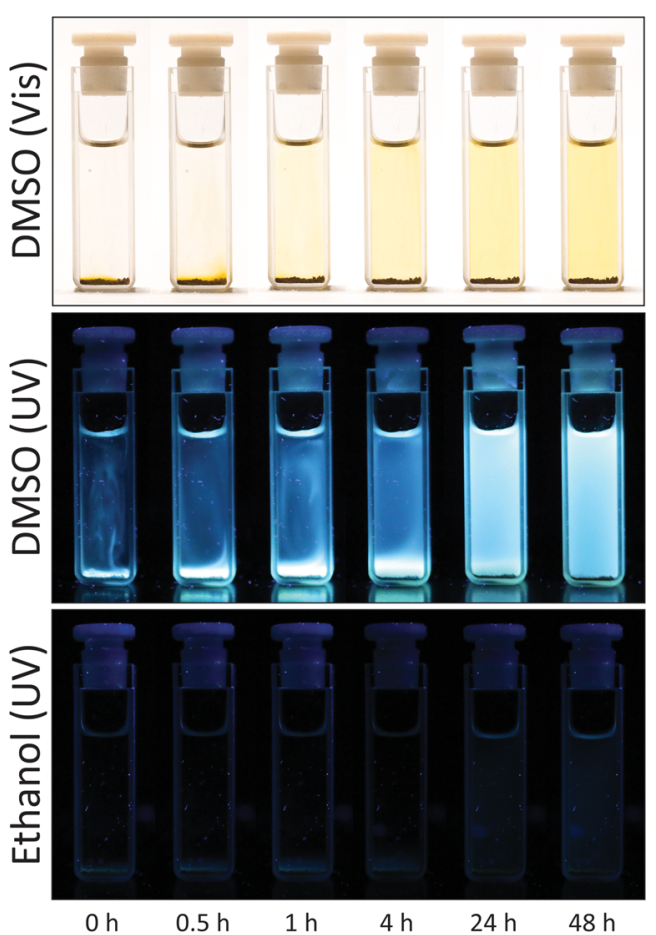

Figure 2. Spontaneous dissolution of PTI-LiBr. Time-lapse photographs of PTI-LiBr following the addition of DMSO or ethanol without disturbing the powder placed at the bottom of the tube and left undisturbed for $48 \mathrm{~h}$. The top panel of images was obtained with visible light and the bottom two panels under long-wavelength $(<365 \mathrm{~nm}) \mathrm{UV}$ illumination.

for any dislocations or point defects. Fast Fourier transform (FFT) analysis of the imaged data (Figure 3d, inset) confirms that the in-plane spacing (unitcell length, $a=0.85 \mathrm{~nm}$ ) corresponds with that of bulk PTI $(a=0.855 \mathrm{~nm}) .^{31}$

To further interrogate the TEM data, image simulations were performed for a combination of defocus and layer thickness conditions, based on the atomic arrangement of the in-plane structure of bulk PTI (SI Section 1, methods). For nanosheets $>1$ monolayer thick, the addition of extra layers only acts to enhance the overall contrast with no change to the structural appearance of the atomic lattice. Therefore, all of the recorded micrographs (e.g., Figure $3 a-c$ and Figure S4) can be identified as few-layer PTI nanosheets. Notably, even for the thinnest examples examined in this study, HRTEM observations and simulations indicate that the voids in the PTI layers maintain the $\mathrm{Br}$ atom site occupancy found in the 3D bulk crystal (Figure 3c, Figure S5).

To further study the morphology of the $2 \mathrm{D}$ nanosheets and their statistical distribution, we used high-speed AFM (HSAFM) to examine populations of the nanosheets deposited onto mica supports from DMF solutions (Figure 3e). The nanosheets have a hexagonal morphology, as expected from the TEM results (Figure 3e, inset), although with reduced vertex sharpness due to AFM-tip convolution effects. The distribution of nanosheet heights above the mica support was automatically extracted for $>2400$ nanosheets together with their corresponding areas (SI Section 1, methods). This analysis confirmed the presence of uniform $2 \mathrm{D}$ objects with heights up to a few nanometers (Figure $3 \mathrm{~h}-\mathrm{j})$. The data reveal that the nanosheets exhibit a range of thicknesses and sheet areas, with maxima close to $\sim 3.2 \mathrm{~nm}$ and $\sim 1500 \mathrm{~nm}^{2}$, respectively. The histogram of nanosheet heights (Figure $3 \mathbf{j}$ ) is fitted by a discrete sum of Gaussian curves (Figure 3j) with its first peak centered at $1.1 \mathrm{~nm}$. The height determined for any monolayer nanosheet above a given substrate is always greater than that expected for the separation between epitaxially stacked multilayers due to interaction effects between the monolayer and its underlying support medium. In the present case, the separation between adjacent PTI sheets ${ }^{31}$ is expected to be $0.35 \mathrm{~nm}$. The first peak in the nanosheet height histogram is 2-3 times greater than this value, so we therefore assign the first peak to a population of bi- or possibly trilayered stacks. However, succeeding peaks within the fitted Gaussian analysis of the height distribution are separated by $0.33 \mathrm{~nm}$, which is very close to the expected layer separation from bulk crystalline PTI-LiBr (Figure $3 i, j)($ ref 31$)$. This analysis gives the modal number of PTI layers per nanosheet stack in the solution to be 8 or 9 . The distribution of sheet diameters determined by both HS-AFM and TEM techniques matches closely that of the starting bulk crystalline materials (Figure 3f). This observation is consistent with gentle delamination and layer dissolution, preserving both the internal structure and external morphology of the carbon nitride nanosheets. The solution-deposited nanosheets have welldefined edges (Figure $3 a-e$ ) indicating that these are not restacked assemblies of nanosheets, but instead have preferentially dissolved in few layer form.

Both bulk and exfoliated carbon nitride materials have been shown to exhibit luminescence in the UV/visible range and they are being explored as potential next-generation materials for light-driven applications including photocatalysis. ${ }^{22-25,37,38}$ Here, we performed steady-state photoluminescence (PL) and PL excitation (PLE) measurements on carbon nitride nanosheets dissolved in DMF. Following UV excitation (260-330 nm), the normalized PL emission spectra exhibit a peak at $\sim 380 \mathrm{~nm}$ that shows little variation in its position, but broadens slightly (fwhm increases from 75 to $124 \mathrm{~nm}$ ) toward the blue-green range for the longest wavelength excitations (Figure 4a). In 2D carbonaceous materials (e.g., graphene oxide sheets and graphitic quantum dots), narrow PL peaks that show little variation with the excitation wavelength are typically associated with highly crystalline, spatially homogeneous emitters over extended length scales. ${ }^{39,40}$ In contrast, PL features that are broad and/or have a strong excitation-wavelength dependence are either associated with large numbers of crystal defects, that behave as independent chromophores with different optical properties. Otherwise broad, dispersive features can indicate wide distributions of nanoparticle sizes in systems where quantum confinement effects cause variations in the electronic band gap. ${ }^{39-43}$ Here we can exclude size-distribution effects within a single sheet since quantum confinement is unlikely, as carbon nitride nanosheets have been proven not to exhibit strong intrasheet electronic charge delocalization ${ }^{44-46}$ and also because of their large lateral dimensions (>50 nm). Quantum confinement between stacked sheets is possible but the height distribution of the dissolved nanosheets, as measured by HS-AFM, is relatively narrow. Therefore, the narrow, when compared to the bulk PTI (Figure S6c, d), wavelength-independent PL signal observed here indicates a low density of crystalline defects.

Charge delocalization between stacked nanosheets has been suggested for heptazine-based carbon nitrides. ${ }^{44,47}$ In a simple model proposed by Merschjann et al., ${ }^{44,47}$ the triazine rings within a single gCN sheet behave as individual, nonconjugated molecules, because they are separated by nonconjugated amine bridges. Their $\pi$-orbitals have little overlap laterally, so charge delocalization is hindered. Stacking multiple sheets in a face-toface configuration, on the other hand, promotes the overlap of 

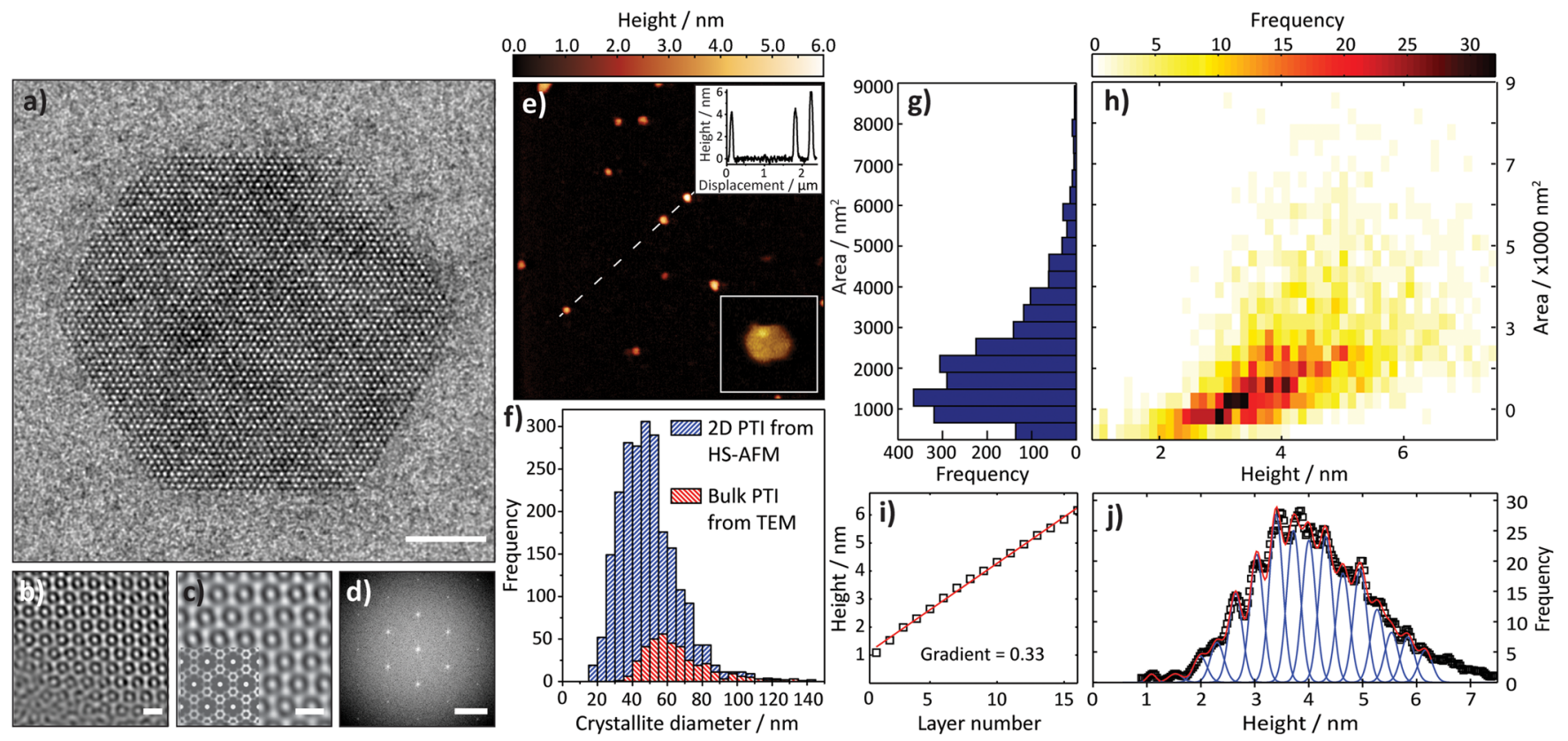

Figure 3. Characterization of PTI·LiBr nanosheets. (a) Filtered TEM image of a few-layer PTI nanosheet from an NMP solution (scalebar $10 \mathrm{~nm}$ ). (b) Higher-magnification micrograph of an edge of the nanosheet shown in (a) at a different defocus condition (scalebar $1 \mathrm{~nm}$ ). (c) Image simulation (bottom left) of the atomic structure of the PTI nanosheet superimposed to the experimental data (see SI Section 1, scalebar $1 \mathrm{~nm}$ ). (d) FFT of image (a) (scalebar $5 \mathrm{~nm}^{-1}$ ) confirming the intralayer structure of the bulk PTI is preserved in nanosheet form. (e) HS-AFM image of few-layer PTI nanosheets deposited from a DMF solution. Top right inset is a line cut across the image as indicated by the dashed line on the image. Bottom right inset shows an AFM image of an individual nanosheet. (f) Histograms showing the crystallite diameter of bulk PTI-LiBr (from analysis of TEM images, red) and the PTI nanosheets taken from HS-AFM (blue). (g) Histogram of sheet areas from $>2400$ crystallites taken from HS-AFM measurements, see SI Section 1, methods. (h) The 2D histogram (color map) of nanosheet areas ( $y$-axis) at different heights ( $x$-axis) from HS-AFM data. (i,j) The 1D height histogram ( $j$ ) (black squares) fitted with a sum of Gaussians (individual Gaussians blue, sum red) and (i) peak heights taken from fit in ( $j$ ) as a function of peak number showing an average peak separation (fitted straight line, red) of $0.33 \mathrm{~nm}$.

the $\pi$-orbitals from isolated triazine groups on adjacent sheets. Therefore, charge movement from one sheet to the next is possible. In order to investigate how the stacking of nanosheets affects their luminescence, we measured the PL for bulk PTI $\mathrm{LiBr}$ crystals, as well as films of aggregated nanosheets deposited from solution. In both cases, the emission spectra are broad with a maximum that is significantly red-shifted compared with PL from the dissolved nanosheets (Figure 4b, Figure S6). The emission for the aggregated samples is centered at $\sim 480 \mathrm{~nm}$ and its fwhm has increased to $\sim 170-200 \mathrm{~nm}$ for both materials. Applying a simple particle in a box model, where charge is assumed to delocalize between sheets and the box size is given by the stack height (see SI Section 2 for details), leads to a semiquantitative interpretation of the PL wavelength dependence on the number of interacting layers in the PTI stack (Figure S7). The calculated emission wavelengths are sensitive to stack thicknesses up to $\sim 40$ layers. The narrow PL signals observed for the nanosheets in solution are consistent with 6-12 average layer thicknesses as found by the HS-AFM measurements. The broad PL observed for the aggregated nanosheet materials, as well as powdered crystalline PTI. LiBr, indicate interactions between stacks extending from between 9 to at least 40 layers in thickness. These observations and calculations demonstrate the tunability of the PL wavelength from narrow UV to broad-band white, depending on the stack thickness.

In summary, we have demonstrated the spontaneous dissolution of crystalline carbon nitride compounds in select polar solvents, yielding solutions of pristine, defect-free, semiconducting 2D nanosheets. The thermodynamically driven dissolution occurs as a result of a free energy gain upon solvent coordination of the nanosheets with respect to that of the isolated PTI crystal and solvent. However, rather than leading to complete delamination into individual monolayers, this dissolution results in a distribution of few-layer stack thicknesses, indicating that the interlayer interactions are energetically similar to those between the solvent and nanosheet surfaces. While our observations suggest that suitable solvents must be polar (Supporting Information Section 2, solvent systems), implying electrostatic interactions with the nanosheets, not all polar solvents solubilize the PTI. $\mathrm{LiBr}$ material, and there is no apparent trend between solubility and dielectric constant for the solvents tried. In the system presented here a full understanding of the factors underpinning favorable solubilization is not expected to be straightforward. Although the formation and stabilization of nanoparticle dispersions have traditionally been analyzed in terms of additive colloidal models, the applicability of such models has recently been called into question. ${ }^{48}$ In particular, it has been noted that local solvent ordering effects become increasingly important upon reduced particle dimensions and that the solvent can no longer be considered as a uniform continuum. ${ }^{48}$ Moreover the factors associated with the local solvent ordering including steric effects, hydrogen bonding and charge-screening are intrinsically interlinked. Indeed significant solvent density enhancement ${ }^{49,50}$ and intricate solvent ordering within nanoparticle solvation shells have been recently measured using advanced $\mathrm{X}$-ray and neutron scattering techniques. ${ }^{49-51}$ In solutions of $\mathrm{C}_{60}$ anions, neutron scattering revealed that the solvent molecules were not arranged so that their dipole moments were directed toward the charged nanoparticle but rather in a way that also maintained the 

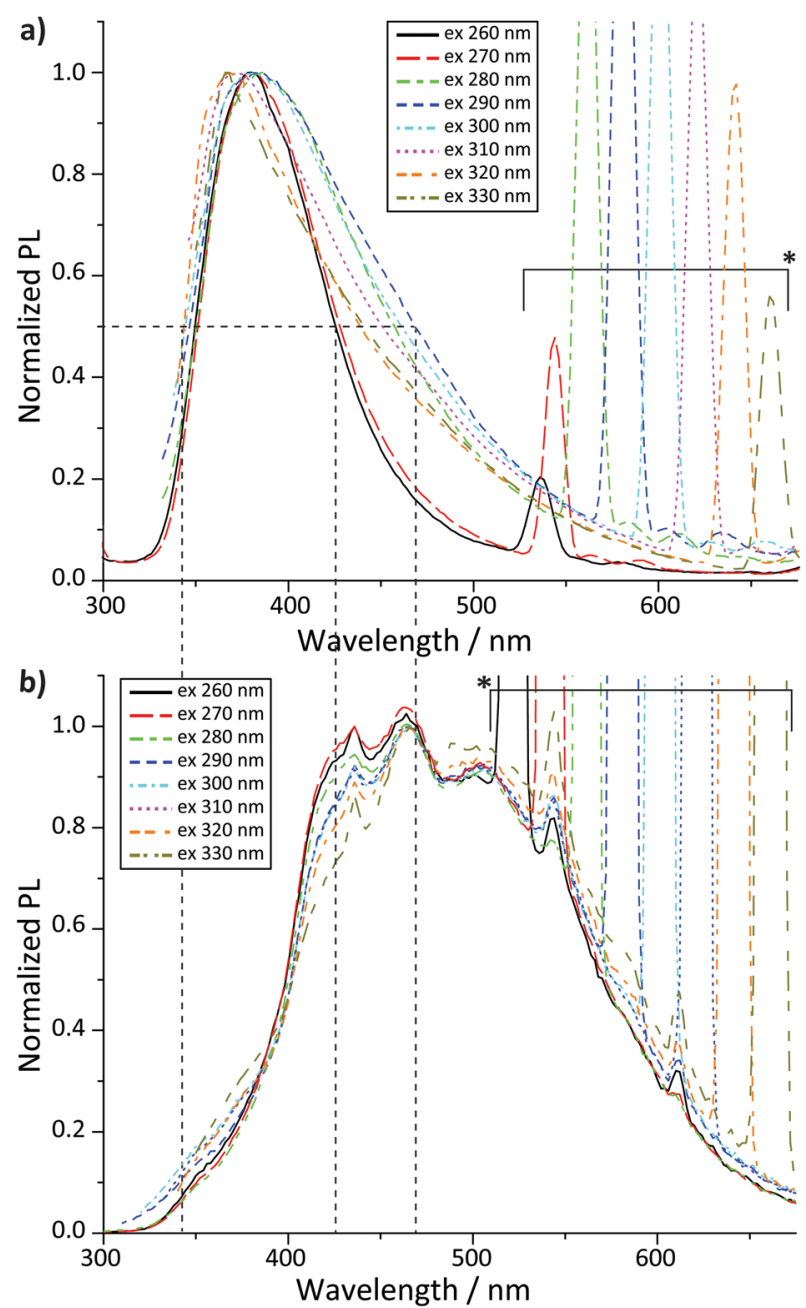

Figure 4. Luminescence properties of hexagonal PTI nanosheets. (a) PL spectra at varying excitation wavelength after spontaneous dissolution in DMF, normalized to the maximum at $\sim 380 \mathrm{~nm}$. (b) PL spectra at varying excitation wavelength of a film deposited from spontaneously dissolved nanosheets, normalized to the maximum at $\sim 450 \mathrm{~nm}$. In all spectra, sharp scattering peaks above $500 \mathrm{~nm}$ are visible (measurement artifacts), located at double the excitation wavelength (indicated by the brackets with asterisks).

intersolvent hydrogen bonding of the bulk solvent within the solvation shells themselves. ${ }^{50,51}$ Similar atomistically resolved measurements are required to fully understand the mechanism for PTI nanosheets dissolution. It is important to note that unlike solutions of clays or ionic solutions of charged 2D materials, ${ }^{15-20}$ the PTI solutes are expected to remain electrically neutral, as $\mathrm{Br}^{-}$ ions are observed to remain incorporated in the dissolved nanosheets.

The well-defined optical properties that result from the highly crystalline, defect-free nanosheets are of practical importance. Carbon nitrides with a less well-ordered heptazine structure are already being developed for photocatalysis applications, and more recently in organic LEDs and solar cells. ${ }^{52-55}$ The tunable emission spectra of crystalline $2 \mathrm{D}$ polytriazine imide nanosheets, dependent on their aggregation state, makes them suitable candidates as UV-blue and white LED emitters. Their wide band gap, and the potential for intersheet coupling and low charge trap density, makes them potential candidates as electron acceptors or charge-selective layers in organic solar cells.
The benefits of spontaneous dissolution as a method for liquid exfoliation are clear: the dissolution process is simple to implement, it is intrinsically scalable, and it results in stable solutions of pristine nanosheets with well-defined functional properties.

\section{ASSOCIATED CONTENT}

Supporting Information

The Supporting Information is available free of charge on the ACS Publications website at DOI: 10.1021/acs.nanolett.7b01353.

Further details of experimental methods. Histogram analysis of bulk PTI crystallites. Time-lapse photographs of PTI $\mathrm{LiBr}$ dissolving in additional solvents. Thermogravimetric analysis of bulk PTI.LiBr and PTI nanosheets solutions. Additional TEM images of PTI nanosheets. Defocus/thickness maps of HR-TEM simulated images of PTI nanosheets. Additional PL and PLE spectra of bulk PTI powder and nanosheet solutions. Further details of the particle in a box model used to validate the intersheet electronic coupling in PTI nanosheets (PDF)

\section{AUTHOR INFORMATION}

\section{Corresponding Authors}

*E-mail: (P.F.M.) p.f.mcmillan@ucl.ac.uk.

*E-mail: (C.A.H.) c.howard@ucl.ac.uk.

ORCID

Christopher A. Howard: 0000-0003-2550-0012

\section{Notes}

The authors declare no competing financial interest.

\section{ACKNOWLEDGMENTS}

This project has received funding from the European Union's Graphene Flagship under Horizon 2020 research and innovation programme Grant Agreement 696656-GrapheneCore1 and from the EPSRC EP/L017091/1. A.M.T. acknowledges the Imperial College Junior Research Fellowship program for funding. The authors would like to thank Furio Corà and Neal Skipper for useful discussions.

\section{REFERENCES}

(1) Nicolosi, V.; Chhowalla, M.; Kanatzidis, M. G.; Strano, M. S.; Coleman, J. N. Science 2013, 340, 1226419.

(2) Ferrari, A. C.; Bonaccorso, F.; Fal'ko, V.; Novoselov, K. S.; Roche, S.; Boggild, P.; Borini, S.; Koppens, F. H. L.; Palermo, V.; Pugno, N.; Garrido, J. A.; Sordan, R.; Bianco, A.; Ballerini, L.; Prato, M.; Lidorikis, E.; Kivioja, J.; Marinelli, C.; Ryhanen, T.; Morpurgo, A.; Coleman, J. N.; Nicolosi, V.; Colombo, L.; Fert, A.; Garcia-Hernandez, M.; Bachtold, A.; Schneider, G. F.; Guinea, F.; Dekker, C.; Barbone, M.; Sun, Z.; Galiotis, C.; Grigorenko, A. N.; Konstantatos, G.; Kis, A.; Katsnelson, M.; Vandersypen, L.; Loiseau, A.; Morandi, V.; Neumaier, D.; Treossi, E.; Pellegrini, V.; Polini, M.; Tredicucci, A.; Williams, G. M.; Hee Hong, B.; Ahn, J. H.; Min Kim, J.; Zirath, H.; van Wees, B. J.; van der Zant, H.; Occhipinti, L.; Di Matteo, A.; Kinloch, I. A.; Seyller, T.; Quesnel, E.; Feng, X.; Teo, K.; Rupesinghe, N.; Hakonen, P.; Neil, S. R. T.; Tannock, Q.; Lofwander, T.; Kinaret, J. Nanoscale 2015, 7 (11), 4598-4810.

(3) Novoselov, K. S.; Geim, A. K.; Morozov, S. V.; Jiang, D.; Zhang, Y.; Dubonos, S. V.; Grigorieva, I. V.; Firsov, A. A. Science 2004, 306 (5696), 666-669.

(4) Withers, F.; Del Pozo-Zamudio, O.; Mishchenko, A.; Rooney, A. P.; Gholinia, A.; Watanabe, K.; Taniguchi, T.; Haigh, S. J.; Geim, A. K.; Tartakovskii, A. I.; Novoselov, K. S. Nat. Mater. 2015, 14 (3), 301-306. 
(5) Lotya, M.; Hernandez, Y.; King, P. J.; Smith, R. J.; Nicolosi, V.; Karlsson, L. S.; Blighe, F. M.; De, S.; Wang, Z.; McGovern, I. T.; Duesberg, G. S.; Coleman, J. N. J. Am. Chem. Soc. 2009, 131 (10), 36113620

(6) Paton, K. R.; Varrla, E.; Backes, C.; Smith, R. J.; Khan, U.; O’Neill, A.; Boland, C.; Lotya, M.; Istrate, O. M.; King, P.; Higgins, T.; Barwich, S.; May, P.; Puczkarski, P.; Ahmed, I.; Moebius, M.; Pettersson, H.; Long, E.; Coelho, J.; O’Brien, S. E.; McGuire, E. K.; Sanchez, B. M.; Duesberg, G. S.; McEvoy, N.; Pennycook, T. J.; Downing, C.; Crossley, A.; Nicolosi, V.; Coleman, J. N. Nat. Mater. 2014, 13 (6), 624-630.

(7) Joensen, P.; Frindt, R. F.; Morrison, S. R. Mater. Res. Bull. 1986, 21 (4), 457-461.

(8) Eda, G.; Yamaguchi, H.; Voiry, D.; Fujita, T.; Chen, M.; Chhowalla, M. Nano Lett. 2011, 11 (12), 5111-5116.

(9) Ganter, P.; Ziegler, C.; Friedrichs, A. T.; Duppel, V.; Scheu, C.; Lotsch, B. V. ChemNanoMat 2017, 3, 411.

(10) Bepete, G.; Anglaret, E.; Ortolani, L.; Morandi, V.; Huang, K.; Pénicaud, A.; Drummond, C. Nat. Chem. 2016, 9 (4), 347-352.

(11) Ou, H.; Lin, L.; Zheng, Y.; Yang, P.; Fang, Y.; Wang, X. Adv. Mater. 2017, 29, 1700008.

(12) Shih, C. J.; Vijayaraghavan, A.; Krishnan, R.; Sharma, R.; Han, J. H.; Ham, M. H.; Jin, Z.; Lin, S.; Paulus, G. L. C.; Reuel, N. F.; Wang, Q. H.; Blankschtein, D.; Strano, M. S. Nat. Nanotechnol. 2011, 6 (7), 439445.

(13) Coleman, J. N.; Lotya, M.; O’Neill, A.; Bergin, S. D.; King, P. J.; Khan, U.; Young, K.; Gaucher, A.; De, S.; Smith, R. J.; Shvets, I. V.; Arora, S. K.; Stanton, G.; Kim, H. Y.; Lee, K.; Kim, G. T.; Duesberg, G. S.; Hallam, T.; Boland, J. J.; Wang, J. J.; Donegan, J. F.; Grunlan, J. C.; Moriarty, G.; Shmeliov, A.; Nicholls, R. J.; Perkins, J. M.; Grieveson, E. M.; Theuwissen, K.; McComb, D. W.; Nellist, P. D.; Nicolosi, V. Science 2011, 331 (6017), 568-571.

(14) Stankovich, S.; Dikin, D. A.; Piner, R. D.; Kohlhaas, K. A.; Kleinhammes, A.; Jia, Y.; Wu, Y.; Nguyen, S. T.; Ruoff, R. S. Carbon 2007, 45 (7), 1558-1565.

(15) Stöter, M.; Rosenfeldt, S.; Breu, J. Annu. Rev. Mater. Res. 2015, 45 (1), 129-151.

(16) Paineau, E.; Philippe, A. M.; Antonova, K.; Bihannic, I.; Davidson, P.; Dozov, I.; Gabriel, J. C. P.; Impéror-Clerc, M.; Levitz, P.; Meneau, F.; Michot, L. J. Liq. Cryst. Rev. 2013, 1 (2), 110-126.

(17) Sposito, G.; Skipper, N. T.; Sutton, R.; Park, S.; Soper, A. K.; Greathouse, J. A. Proc. Natl. Acad. Sci. U. S. A. 1999, 96 (7), 3358-3364.

(18) Vallés, C.; Drummond, C.; Saadaoui, H.; Furtado, C. A.; He, M.; Roubeau, O.; Ortolani, L.; Monthioux, M.; Pénicaud, A. J. Am. Chem. Soc. 2008, 130 (47), 15802-15804.

(19) Milner, E. M.; Skipper, N. T.; Howard, C. A.; Shaffer, M. S. P.; Buckley, D. J.; Rahnejat, K. A.; Cullen, P. L.; Heenan, R. K.; Lindner, P.; Schweins, R. J. Am. Chem. Soc. 2012, 134 (20), 8302-8305.

(20) Cullen, P. L.; Cox, K. M.; Bin Subhan, M. K.; Picco, L.; Payton, O. D.; Buckley, D. J.; Miller, T. S.; Hodge, S. A.; Skipper, N. T.; Tileli, V.; Howard, C. A. Nat. Chem. 2016, 9, 244-249.

(21) McDermott, E. J.; Wirnhier, E.; Schnick, W.; Virdi, K. S.; Scheu, C.; Kauffmann, Y.; Kaplan, W. D.; Kurmaev, E. Z.; Moewes, A. J. Phys. Chem. C 2013, 117 (17), 8806-8812.

(22) Thomas, A.; Fischer, A.; Goettmann, F.; Antonietti, M.; Muller, J. O.; Schlogl, R.; Carlsson, J. M. J. Mater. Chem. 2008, 18 (41), 48934908.

(23) Liu, J.; Liu, Y.; Liu, N.; Han, Y.; Zhang, X.; Huang, H.; Lifshitz, Y.; Lee, S. T.; Zhong, J.; Kang, Z. Science 2015, 347 (6225), 970-974.

(24) Wang, Y.; Wang, X.; Antonietti, M. Angew. Chem., Int. Ed. 2012, $51(1), 68-89$.

(25) Schwinghammer, K.; Mesch, M. B.; Duppel, V.; Ziegler, C.; Senker, J.; Lotsch, B. V. J. Am. Chem. Soc. 2014, 136 (5), 1730-1733.

(26) Lin, L.; Ou, H.; Zhang, Y.; Wang, X. ACS Catal. 2016, 6 (6), 3921-3931.

(27) Mansor, N.; Jorge, A. B.; Corà, F.; Gibbs, C.; Jervis, R.; McMillan, P. F.; Wang, X.; Brett, D. J. L. J. Phys. Chem. C 2014, 118 (13), 68316838.

(28) Mansor, N.; Miller, T. S.; Dedigama, I.; Jorge, A. B.; Jia, J.; Brázdová, V.; Mattevi, C.; Gibbs, C.; Hodgson, D.; Shearing, P. R.;
Howard, C. A.; Corà, F.; Shaffer, M.; Brett, D. J. L.; McMillan, P. F. Electrochim. Acta 2016, 222, 44-57.

(29) Miller, T. S.; Jorge, A. B.; Suter, T. M.; Sella, A.; Corà, F.; McMillan, P. F. Phys. Chem. Chem. Phys. 2017, 19, 15613.

(30) Bojdys, M. J.; Müller, J. O.; Antonietti, M.; Thomas, A. Chem. Eur. J. 2008, 14 (27), 8177-8182.

(31) Wirnhier, E.; Döblinger, M.; Gunzelmann, D.; Senker, J.; Lotsch, B. V.; Schnick, W. Chem. - Eur. J. 2011, 17 (11), 3213-3221.

(32) Rong, M.; Lin, L.; Song, X.; Wang, Y.; Zhong, Y.; Yan, J.; Feng, Y.; Zeng, X.; Chen, X. Biosens. Bioelectron. 2015, 68, 210-217.

(33) Zhang, X.; Xie, X.; Wang, H.; Zhang, J.; Pan, B.; Xie, Y. J. Am. Chem. Soc. 2013, 135 (1), 18-21.

(34) Bojdys, M. J.; Severin, N.; Rabe, J. P.; Cooper, A. I.; Thomas, A.; Antonietti, M. Macromol. Rapid Commun. 2013, 34 (10), 850-854.

(35) Chong, S. Y.; Jones, J. T. A.; Khimyak, Y. Z.; Cooper, A. I.; Thomas, A.; Antonietti, M.; Bojdys, M. J. J. Mater. Chem. A 2013, 1 (4), $1102-1107$

(36) Zhang, Y.; Thomas, A.; Antonietti, M.; Wang, X. J. Am. Chem. Soc. 2009, 131 (1), 50-51.

(37) Wang, X.; Maeda, K.; Thomas, A.; Takanabe, K.; Xin, G.; Carlsson, J. M.; Domen, K.; Antonietti, M. Nat. Mater. 2009, 8 (1), 7680.

(38) Su, F.; Mathew, S. C.; Lipner, G.; Fu, X.; Antonietti, M.; Blechert, S.; Wang, X. J. Am. Chem. Soc. 2010, 132 (46), 16299-16301.

(39) Gan, Z.; Xu, H.; Hao, Y. Nanoscale 2016, 8 (15), 7794-7807.

(40) Kozák, O.; Sudolská, M.; Pramanik, G.; Cígler, P.; Otyepka, M.; Zbořil, R. Chem. Mater. 2016, 28 (12), 4085-4128.

(41) Yeh, T.; Teng, C.; Chen, L.; Chen, S.; Teng, H. J. Mater. Chem. A 2016, 4 (6), 2014-2048.

(42) Li, H.; Shao, F. Q.; Huang, H.; Feng, J. J.; Wang, A. J. Sens. Actuators, B 2016, 226, 506-511.

(43) Liu, S.; Tian, J.; Wang, L.; Luo, Y.; Zhai, J.; Sun, X. J. Mater. Chem. 2011, 21 (32), 11726-11729.

(44) Merschjann, C.; Tyborski, T.; Orthmann, S.; Yang, F.; Schwarzburg, K.; Lublow, M.; Lux-Steiner, M. C.; Schedel-Niedrig, T. Phys. Rev. B: Condens. Matter Mater. Phys. 2013, 87 (20), 205204.

(45) Melissen, S.; Le Bahers, T.; Steinmann, S. N.; Sautet, P. J. Phys.

Chem. C 2015, 119 (45), 25188-25196.

(46) Huda, M. N.; Turner, J. A. J. Appl. Phys. 2010, 107 (12), 123703.

(47) Merschjann, C.; Tschierlei, S.; Tyborski, T.; Kailasam, K.; Orthmann, S.; Hollmann, D.; Schedel-Niedrig, T.; Thomas, A.; Lochbrunner, S. Adv. Mater. 2015, 27 (48), 7993-7999.

(48) Silvera Batista, C. A.; Larson, R. G.; Kotov, N. A. Science 2015, 350,1242477

(49) Zobel, M.; Neder, R. B.; Kimber, S. A. J. Science 2015, 347 (6219), 292.

(50) Howard, C. A.; Thompson, H.; Wasse, J. C.; Skipper, N. T. J. Am. Chem. Soc. 2004, 126 (41), 13228-13229.

(51) Howard, C. A.; Wasse, J. C.; Skipper, N. T.; Thompson, H.; Soper, A. K. J. Phys. Chem. C 2007, 111 (15), 5640-5647.

(52) Xu, J.; Herraiz-Cardona, I.; Yang, X.; Gimenez, S.; Antonietti, M.; Shalom, M. Adv. Opt. Mater. 2015, 3 (8), 1052-1058.

(53) Zhou, L.; Xu, Y.; Yu, W.; Guo, X.; Yu, S.; Zhang, J.; Li, C. J. Mater. Chem. A 2016, 4 (21), 8000-8004.

(54) Lee, W.; Jun, Y.; Park, J.; Stucky, G. D. J. Mater. Chem. A 2015, 3 (48), 24232-24236.

(55) Bayan, S.; Gogurla, N.; Midya, A.; Ray, S. K. Carbon 2016, 108, $335-342$. 